\title{
Drug-specific laterality effects on frontal lobe activation of atomoxetine and methylphenidate in attention deficit hyperactivity disorder boys during working memory
}

Cubillo, Ana ; Smith, Anna B ; Barrett, Nadia ; Giampietro, Vincent ; Brammer, Michael ; Simmons, Andrew ; Rubia, Katya

\begin{abstract}
Background The catecholamine reuptake inhibitors methylphenidate (MPH) and atomoxetine (ATX) are the most common treatments for attention deficit hyperactivity disorder (ADHD). This study compares the neurofunctional modulation and normalization effects of acute doses of MPH and ATX within medication-naive ADHD boys during working memory (WM). Method A total of 20 medicationnaive ADHD boys underwent functional magnetic resonance imaging during a parametric WM n-back task three times, under a single clinical dose of either MPH, ATX or placebo in a randomized, doubleblind, placebo-controlled, cross-over design. To test for normalization effects, brain activations in ADHD under each drug condition were compared with that of 20 age-matched healthy control boys. Results Relative to healthy boys, ADHD boys under placebo showed impaired performance only under high WM load together with significant underactivation in the bilateral dorsolateral prefrontal cortex (DLPFC). Both drugs normalized the performance deficits relative to controls. ATX significantly enhanced right DLPFC activation relative to MPH within patients, and significantly normalized its underactivation relative to controls. MPH, by contrast, both relative to placebo and ATX, as well as relative to controls, upregulated the left inferior frontal cortex (IFC), but only during 2-back. Both drugs enhanced frontotemporo-striatal activation in ADHD relative to control boys and deactivated the default-mode network, which were negatively associated with the reduced DLPFC activation and performance deficits, suggesting compensation effects. Conclusions The study shows both shared and drug-specific effects. ATX upregulated and normalized right DLPFC underactivation, while MPH upregulated left IFC activation, suggesting drug-specific laterality effects on prefrontal regions mediating WM.
\end{abstract}

DOI: https://doi.org/10.1017/S0033291713000676

Posted at the Zurich Open Repository and Archive, University of Zurich

ZORA URL: https://doi.org/10.5167/uzh-100568

Journal Article

Originally published at:

Cubillo, Ana; Smith, Anna B; Barrett, Nadia; Giampietro, Vincent; Brammer, Michael; Simmons, Andrew; Rubia, Katya (2014). Drug-specific laterality effects on frontal lobe activation of atomoxetine and methylphenidate in attention deficit hyperactivity disorder boys during working memory. Psychological Medicine, 44(03):633-646.

DOI: https://doi.org/10.1017/S0033291713000676 


\title{
Drug-specific laterality effects on frontal lobe activation of atomoxetine and methylphenidate in attention deficit hyperactivity disorder boys during working memory
}

\author{
A. Cubillo ${ }^{1}+$, A. B. Smith ${ }^{1} t$, N. Barrett ${ }^{1}$, V. Giampietro ${ }^{2}$, M. Brammer ${ }^{2}$, A. Simmons Br,3 $^{2,4}$ \\ and K. Rubia ${ }^{1 *}$ \\ ${ }^{1}$ Department of Child and Adolescent Psychiatry, Institute of Psychiatry, King's College London, London, UK \\ ${ }^{2}$ Department of Neuroimaging, Institute of Psychiatry, King's College London, London, UK \\ ${ }^{3}$ Centre for Neurodegeneration Research, Institute of Psychiatry, King's College London, London, UK \\ ${ }^{4}$ NIHR Biomedical Research Centre for Mental Health at South London and Maudsley NHS Trust and Institute of Psychiatry, King's College London, \\ London, UK
}

Background. The catecholamine reuptake inhibitors methylphenidate (MPH) and atomoxetine (ATX) are the most common treatments for attention deficit hyperactivity disorder (ADHD). This study compares the neurofunctional modulation and normalization effects of acute doses of MPH and ATX within medication-naive ADHD boys during working memory (WM).

Method. A total of 20 medication-naive ADHD boys underwent functional magnetic resonance imaging during a parametric WM n-back task three times, under a single clinical dose of either MPH, ATX or placebo in a randomized, doubleblind, placebo-controlled, cross-over design. To test for normalization effects, brain activations in ADHD under each drug condition were compared with that of 20 age-matched healthy control boys.

Results. Relative to healthy boys, ADHD boys under placebo showed impaired performance only under high WM load together with significant underactivation in the bilateral dorsolateral prefrontal cortex (DLPFC). Both drugs normalized the performance deficits relative to controls. ATX significantly enhanced right DLPFC activation relative to MPH within patients, and significantly normalized its underactivation relative to controls. MPH, by contrast, both relative to placebo and ATX, as well as relative to controls, upregulated the left inferior frontal cortex (IFC), but only during 2-back. Both drugs enhanced fronto-temporo-striatal activation in ADHD relative to control boys and deactivated the default-mode network, which were negatively associated with the reduced DLPFC activation and performance deficits, suggesting compensation effects.

Conclusions. The study shows both shared and drug-specific effects. ATX upregulated and normalized right DLPFC underactivation, while MPH upregulated left IFC activation, suggesting drug-specific laterality effects on prefrontal regions mediating WM.

Received 16 February 2012; Revised 16 January 2013; Accepted 5 March 2013; First published online 19 April 2013

Key words: Atomoxetine, attention deficit hyperactivity disorder, functional magnetic resonance imaging, methylphenidate, n-back, working memory.

\section{Introduction}

Attention deficit hyperactivity disorder (ADHD) is characterized by age-inappropriate symptoms of inattention, impulsivity and hyperactivity (APA, 2000). One of the key neuropsychological deficits is in working memory (WM) (Martinussen et al. 2005), defined as

* Address for correspondence: K. Rubia, Ph.D., Department of Child and Adolescent Psychiatry/SGDP, P046, Institute of Psychiatry, 16 De Crespigny Park, London SE5 8AF, UK.

(Email: katya.rubia@kcl.ac.uk)

+ These authors contributed equally as joint first authors. the temporary storage and manipulation of information (Baddeley, 1996).

Children with ADHD have consistently shown deficits in verbal-auditory and visuo-spatial WM tasks (Rommelse et al. 2008; Toplak et al. 2008; Gau \& Shang, 2010; Rhodes et al. 2011; for meta-analyses, see Martinussen et al. 2005 and Willcutt et al. 2005) including visual-object (Pasini et al. 2007) and phonological n-back tasks (Klein et al. 2006; Pasini et al. 2007; Kobel et al. 2009). These deficits have been shown to be underpinned by functional magnetic resonance imaging (fMRI) evidence for reduced fronto-striatal and temporo-parietal activation during visuo-spatial (Silk 
et al. 2005; Vance et al. 2007) and verbal WM tasks (Kobel et al. 2009).

The stimulant methylphenidate (MPH) and the nonstimulant atomoxetine (ATX) are the most commonly prescribed pharmacological treatments for ADHD, showing comparable efficacy in $65-70 \%$ of cases (Hazell et al. 2010). However, the drug-specific mechanisms of action on brain function in ADHD patients are unknown.

$\mathrm{MPH}$ blocks dopamine (DA) transporters in the striatum (Volkow et al. 1998) and norepinephrine (NE) transporters (NET) in NET-rich regions including prefrontal regions, where it enhances both catecholamines (Hannestad et al. 2010). ATX is a selective pre-synaptic NET blocker affecting NE and DA in the prefrontal cortex (Bymaster et al. 2002) and NE in the thalamus, locus coeruleus and cerebellum, with minimal striatal effects (Gallezot et al. 2011).

Placebo-controlled studies in medication-naive ADHD patients showed that a single dose of MPH upregulated and/or normalized the fronto-striatal, temporoparietal and cerebellar underactivations during cognitive control tasks (Rubia et al. 2009a,b, 2011a,b), with similar effects being reported in previously medicated children compared with off-medication status (Vaidya et al. 1998; Shafritz et al. 2004; Epstein et al. 2007).

Few fMRI studies, however, have tested for $\mathrm{MPH}$ effects on WM in ADHD children, with inconsistent results. Thus, in previously medicated ADHD children compared with controls, MPH normalized the inferior and medial frontal cortices (IFC/MFC) and striatal underactivation during a delayed matched to sample task (Prehn-Kristensen et al. 2011) and upregulated activation and functional integration in fronto-parietal WM networks during a Sternberg WM task (Wong \& Stevens, 2012). However, a single dose of MPH downregulated MFC and parietal activation during a delayed WM match-to-sample task in female ADHD subjects (Sheridan et al. 2010) or had no effects in ADHD boys (Kobel et al. 2009). These studies, however, did not include a placebo condition, had small sample sizes and recruited patients with a previous history of stimulant medication, shown to affect both brain activation and structure (Nakao et al. 2011; Hart et al. 2012, 2013).

In the only placebo-controlled cross-over study comparing the effects of single-dose challenges of $\mathrm{MPH}$ and ATX in medication-naive children with ADHD, MPH showed drug-specific upregulation and normalization effects during motor response inhibition in the right IFC and cerebellum, while both drugs normalized left IFC underactivation (Cubillo et al. 2012b).

However, no fMRI study has as yet investigated the effects of ATX on brain activation in ADHD patients during WM, or compared its effects with those of
MPH. In healthy adults, acute ATX administration upregulates the IFC and superior temporal gyrus (STG) activation during inhibitory tasks (Chamberlain et al. 2009; Graf et al. 2011), and using multivariate pattern recognition analyses ATX had relatively stronger deactivation effects on the default-mode network (DMN) while MPH has relatively stronger upregulating effects on WM networks (Marquand et al. 2011).

We therefore conducted a randomized, doubleblind, placebo-controlled, cross-over pharmacological fMRI study to test for drug-specific effects of a single clinical dose of either MPH or ATX on brain activation of medication-naive boys with ADHD during a WM task. We chose a parametric verbal WM task, given: (a) the impact of verbal WM deficits on academic performance; $(b)$ prior evidence for impairments in this task in ADHD children (Martinussen et al. 2005; Willcutt et al. 2005; Pasini et al. 2007; Kobel et al. 2009); and (c) evidence for more pronounced impairments in ADHD with increasing WM load (Goldberg et al. 2005; Kobel et al. 2009; Gau \& Shang, 2010). The focus on single rather than long-term drug effects on neurofunctional mechanisms avoids potential confounds including side effects, symptomatic improvement or chronic effects on brain activation. We furthermore compared brain activation during the WM task in patients under each drug condition with that of age-matched healthy controls to test for potential drug normalization effects. Based on previous evidence on the n-back task, we hypothesized that ADHD patients would be more impaired with increasing WM load (Kobel et al. 2009). Based on previous fMRI studies of WM in ADHD children (Prehn-Kristensen et al. 2011), we hypothesized that ADHD boys would show underactivation relative to controls in dorsolateral prefrontal cortex (DLPFC) and parietal regions. Furthermore, based on previous findings of frontostriatal upregulation by single doses of $\mathrm{MPH}$ of brain activation in ADHD (Vaidya et al. 1998; Shafritz et al. 2004; Rubia et al. 2009a, 2011a,b; Prehn-Kristensen et al. 2011) and of ATX on frontal brain activation in healthy adults (Chamberlain et al. 2009; Graf et al. 2011), we hypothesized that both drugs would upregulate and normalize the reduced activation in the lateral PFC and that MPH would additionally enhance basal ganglia activation.

\section{Method}

\section{Subjects}

A total of 20 medication-naive right-handed boys (aged 10-17 years) were recruited from clinics who had a clinical diagnosis of ADHD, inattentive/hyperactive-impulsive combined subtype, as assessed by 
an experienced child psychiatrist using the standardized Maudsley diagnostic interview that assesses ADHD according to Diagnostic and Statistical Manual of Mental Disorders, 4th edition, Text Revision (DSM-IV-TR) criteria and is of standard use in the UK (Goldberg \& Murray, 2002). ADHD boys scored above the clinical threshold for hyperactiveimpulsive/inattentive symptoms on the Strengths and Difficulties Questionnaire for parents (SDQ; Goodman \& Scott, 1999), the Conners' Parent Rating Scale (CPRS-R; Conners et al. 1998), and below the clinical threshold on the Social Communication Questionnaire (SCQ; Rutter et al. 2003) (Supplementary Table 1). Patients were scanned in a double-blind, placebo-controlled, cross-over design. On each scanning session, they received a single dose of either placebo (vitamin C: $50 \mathrm{mg}$ ), MPH (Equasym: $0.3 \mathrm{mg} / \mathrm{kg}$, range $5-20 \mathrm{mg}$ ) or ATX (Strattera: $1 \mathrm{mg} / \mathrm{kg}$, range $16-66 \mathrm{mg}$ ), in a pseudo-randomized order, and remained medication-free between scans. A Latin square design was used for the randomization to achieve counterbalanced effects. Dosages were determined following NICE guidelines of clinical efficacious dosages with minimal side effects at the time of the study (National Institute for Heath and Clinical Excellence, 2008). Based on pharmacokinetic evidence, both medications were administered $1.5 \mathrm{~h}$ before the scan to allow for maximum absorption (Chan et al. 1983; Witcher et al. 2003). The same or similar dosages and time lapses between drug administration and scan have shown to be sufficient to observe changes in brain activation and performance in ADHD patients (MPH) (Lijffijt et al. 2006; Rubia et al. 2011a,b) and healthy controls (ATX) (Chamberlain et al. 2007, 2009). All three medications were over-encapsulated using the same capsules by the pharmacist.

A total of 20 right-handed healthy boys (aged 10-17 years) were recruited through advertisement in the same geographical South London area of the UK. They scored below clinical cut-offs for the SDQ, SCQ and CPRS-R. They were scanned once, unmedicated.

Exclusion criteria for all participants were an intelligence quotient (IQ) below 70 on the Wechsler Intelligence Scale for Children, 4th version (WISC-IV; Wechsler, 2004) (administered in the clinics: $n=7$ ) or in the Wechsler Abbreviated Scale of Intelligence (Wechsler, 1999) (administered in our laboratory: $n=13$ ), history of substance abuse or neurological deficits, presence of psychiatric disorders (except for ADHD and conduct disorder/oppositional defiant disorder in the ADHD group: $n=2$ ), learning disability, and reading, speech or language disorder.

One-way analyses of variance (ANOVAs) showed no between-group differences for age $\left(F_{1,38}=0.88\right.$, $p<0.35)$ but did so for IQ $\left(F_{1,38}=42, \quad p<0.001\right)$
(Supplementary Table 1). Low IQ is associated with ADHD (Bridgett \& Walker, 2006). Although commonly conducted in case-control studies, analysis of covariance is statistically illegitimate when the covariate is an attribute of the disorder and when groups were not randomly selected (Evans \& Anastasio, 1968; Miller \& Chapman, 2001; Dennis et al. 2009). Furthermore, WM has been shown to be closely associated with IQ and is part of the WISC-IV (Wechsler, 2004). Therefore, co-varying for IQ during this task would in fact mean co-varying for any potential group differences in WM. Consequently, all analyses were conducted without IQ as a covariate. However, in order to test for potential effects of outliers, we repeated the fMRI analyses excluding subjects with an IQ more than 2 s.D. above or below average (i.e. four subjects in each group).

Participants were paid $£ 50$ for each scanning session. Parental/child informed consent/assent and approval from the local ethical committee were obtained.

\section{WM task (n-back)}

Subjects practised the task once before scanning. The 6-min block design WM task (Ginestet \& Simmons, 2011) consists of four conditions. During '1-back', '2-back' and '3-back' conditions, subjects are presented with series of letters $(\mathrm{A}-\mathrm{Z})$ ( $1 \mathrm{~s}$ duration, inter-trial interval=2s) and must respond with their right thumb using a button box whenever the letter presented is the same as one, two or three before it, respectively (e.g. 2-back: $\mathrm{B} / \mathrm{J} / \mathrm{A} / \mathrm{J}$ ). This requires both storage and continuous updating of stimuli being held in WM. In the baseline vigilance '0-back' condition, subjects must respond to each $X$ that appears on the screen. The task consists of 12 randomized blocks. Before each block, written instructions of $3 \mathrm{~s}$ duration are shown as to which condition is next (i.e. '0-back', 1-back', etc.). In each of the WM blocks of $30 \mathrm{~s}$ duration only one WM condition is presented (i.e. 2-back), and contains 15 stimuli: three targets and 12 non-targets. Each condition is presented three times. Performance data were recorded during scanning. The dependent variable is accuracy (percentage of correctly identified targets).

\section{fMRI acquisition and analyses}

Gradient-echo echoplanar MR imaging (EPI) data were acquired on a GE Signa 3 T Horizon HDx system (General Electric, USA) at the Centre for Neuroimaging Sciences, Institute of Psychiatry, King's College London, UK. A semi-automated quality-control procedure ensured consistent image quality (Simmons et al. 1999). A quadrature birdcage head coil was used for radio frequency transmission and reception. 
Table 1. Performance data for 20 healthy control boys and 20 boys with ADHD under each medication condition

\begin{tabular}{llllll}
\hline Performance variable & Task condition & Controls & ADHD placebo & ADHD MPH & ADHD ATX \\
\hline Accuracy (\% correct responses) & 0-back & $96(11)$ & $98(4)$ & $99(3)$ & $97(8)$ \\
& 1-back & $92(17)$ & $97(6)$ & $98(6)$ & $95(16)$ \\
& 2-back & $87(22)$ & $77(26)$ & $82(25)$ & $81(26)$ \\
& 3-back & $68(21)$ & $60(28)$ & $66(24)$ & $63(27)$ \\
\hline
\end{tabular}

ADHD, Attention deficit hyperactivity disorder; MPH, methylphenidate; ATX, atomoxetine.

In each of 39 non-contiguous planes parallel to the anterior-posterior commissure line, $186 \mathrm{~T}_{2}{ }^{*}$-weighted MR images depicting blood oxygen level-dependent (BOLD) contrasts covering the whole brain were acquired (echo time $=30 \mathrm{~ms}$, repetition time $=2 \mathrm{~s}$, flip angle $=75^{\circ}$, in-plane resolution $=3 \mathrm{~mm}$, slice thickness $=3.5 \mathrm{~mm}$, slice-skip $=0.5 \mathrm{~mm}$ ). This EPI dataset provided complete brain coverage.

Blocked fMRI data were acquired in randomized block presentation, and analysed using the nonparametric XBAM software (Brammer et al. 1997; Bullmore et al. 1999, 2001; Brain Image Analysis Unit, 2011). Methods for individual fMRI analyses and group mapping are described in the Supplementary text. For between-group comparisons, a $2 \times 3$ split plotdesign ANOVA (groups: controls, patients; WM load: 1-back, 2-back, 3-back, each separately contrasted with 0-back) was conducted. For within-group comparisons, a $3 \times 3$ factorial-design repeated-measures ANOVA (drug condition: placebo, MPH, ATX; WM load: 1-back, 2-back, 3-back, separately contrasted with 0-back) was conducted. Statistical measures of BOLD response were extracted for each participant in each of the clusters of activation differences for each of the three contrasts, and post hoc analyses were conducted to clarify the direction of the differences. Within patients, repeated-measures ANOVAs on the extracted BOLD response measures were conducted to test for potential order effects.

\section{Performance analysis}

For the main performance measure of accuracy within patients, repeated-measures ANOVAs were conducted with drug condition (placebo, MPH, ATX) and WM load (0-back, 1-back, 2-back, 3-back) as within-subject factors. For case-control comparisons, three repeatedmeasures ANOVAs (controls versus ADHD under placebo; controls versus ADHD under MPH and controls versus ADHD under ATX) were conducted with WM load as the within-subjects factor and group as the between-subjects factor. Repeated-measures ANOVAs were used to test for potential scan order effects.

\section{Results}

\section{Performance data}

Across all participants, there was a significant WM load effect in accuracy $\left(F_{3,114}=49, p<0.001\right)$, showing that accuracy decreased with increasing WM load (Table 1).

In case-control comparisons, no group effects were found. However, as hypothesized, there was a significant interaction effect between WM load and group in accuracy when healthy controls were compared with patients under placebo $\left(F_{3,114}=3, p<0.035\right)$ due to patients being more accurate during 0-back and 1-back but less accurate during 2- and 3-back than healthy controls (Table 1).

Within patients, no effects of drug condition or WM load $\times$ drug condition were detected on performance variables.

No other group, group $\times \mathrm{WM}$ load interaction or drug order effects were observed.

\section{Brain activation}

Motion

Participants were excluded if they had $>3 \mathrm{~mm}$ in the $X$ or $\mathrm{Y}$ dimensions. Multivariate ANOVA showed no significant group differences between controls and ADHD patients under each drug condition in the extent of mean rotation and translation movement parameters in the three-dimensional Euclidean space $\left(F_{6,152}=1, p=0.43\right)$.

\section{Within-group brain activations}

Within-group brain activations are shown in Supplementary Fig. 1 and described in the Supplementary text and Supplementary Table 2.

\section{ANOVA between-group comparisons between healthy controls and ADHD boys under placebo, MPH or ATX}

Placebo

Controls compared with patients under placebo showed enhanced activation in the bilateral DLPFC 
(Table 2, Fig. 1a). There was no enhanced activation in patients compared with healthy controls.

Given that patients were less accurate than controls in the 2- and 3-back conditions, we hypothesized that accuracy would be positively correlated with DLPFC activation in these conditions. Hence, one-tailed Pearson correlations were conducted in both groups separately between statistical measures of the BOLD response in the left and right DLPFC and a composite score of their accuracy during 2- and 3-back. At a trend level, activation in the right DLPFC was correlated positively with accuracy within controls $(r=0.35, p<$ 0.06). No correlations were observed for left DLPFC activation.

\section{$\mathrm{MPH}$}

After the single dose of MPH, patients compared with controls showed underactivation in the same left and right DLPFC clusters as under placebo. However, ADHD patients relative to controls showed additional, enhanced activation in a cluster comprising the right STG/premotor cortex, striatum/thalamus, insula, reaching into the cerebellar vermis (Table 2, Fig. 1a). To test whether the increased right medial fronto-STG-striatal activation in patients under MPH was compensatory for the reduced bilateral DLPFC activation, statistical measures of BOLD response were extracted for each patient in these three clusters and correlated with the DLPFC activation clusters as well as with the accuracy composite score. Pearson correlations showed that the medial fronto-STG-striatal activation was negatively correlated with the left DLPFC activation $(r=-0.5, p<0.012)$ and positively with accuracy $(r=$ $0.37, p<0.05)$.

\section{ATX}

Under the single dose of ATX, patients compared with controls still showed reduced activation in the left DLPFC. However, they no longer showed underactivation of the right DLPFC. In addition, abnormally enhanced activation was observed in ADHD relative to controls in a right-lateralized cluster of IFC and STG, insula, thalamus and striatum (Table 2, Fig. 1a), which within patients was positively correlated with accuracy $(r=0.54, p<0.007)$.

Repeated-measures ANOVA within patients, for all three contrasts, showed no significant scan order effects on the extracted BOLD response measures.

When those subjects whose IQ scores were more than 2 s.D. above or below average were excluded, all findings remained, although at a more lenient $p$ value $(p<0.03)$.
Effect size comparisons of case-control conditions to test for significant 'normalization' effects

To establish whether the group differences between controls and patients under each drug condition were significantly different, we directly compared the effect sizes (ES) of the group differences from the three casecontrol comparisons (Matthews \& Altman, 1996) (see details in the Supplementary text).

In the right DLPFC, the ES of the case-control contrast under ATX was significantly different from the ES of the case-control comparison under $\mathrm{MPH}(p<$ 0.03), suggesting that ATX significantly enhanced activation in this region relative to $\mathrm{MPH}$. For the cluster of enhanced right fronto-STG-striatum activation under $\mathrm{MPH}$ relative to controls the ES of the case-control contrast under MPH was significantly higher than the ES of the case-control comparisons under placebo $(p<$ $0.004)$ and ATX $(p<0.05)$. Finally, for the cluster of enhanced right IFC-STG-striato-thalamic activation under ATX relative to controls, the ES was significantly larger for the case-control contrast under ATX relative to those of the case-control comparisons under placebo $(p<0.007)$ and under MPH $(p<0.03)$ (Supplementary Table 3).

\section{ANOVA within-patient comparison between placebo, MPH and ATX}

Given the reduced activation in the left and right DLPFC, we tested for significant upregulation effects of both drugs in the frontal cortex. The Talairach Client (Lancaster et al. 1997, 2000) was used to define a mask of the frontal lobe, restricting the analysis to those voxels present in the mask. A significant effect of drug condition was observed in a cluster in the right DLPFC [13 voxels, peak Talairach coordinates $(\mathrm{x}, \mathrm{y}, \mathrm{z}): 25,44,26$; Brodmann area (BA) 10/9, $p<$ $0.044]$, which was due to significantly enhanced activation when under ATX relative to MPH $(p<0.002)$, as well as when under placebo relative to $\mathrm{MPH}(p<$ 0.01) (Fig. 1b). There were no significant correlations between brain activation changes and performance.

\section{Interaction effects}

Between-groups comparisons: WM load $\times$ group

No significant interaction effects were observed in the comparison between healthy controls and patients under placebo.

In the comparison between healthy and ADHD boys under $\mathrm{MPH}$, a significant interaction of group $\times \mathrm{WM}$ load was observed in the left IFC reaching into the DLPFC, putamen and anterior insula, due to enhanced activation in ADHD under MPH relative to controls during 2-back $(p<0.01)$. Additional interaction effects 
(a)

Controls versus ADHD placebo
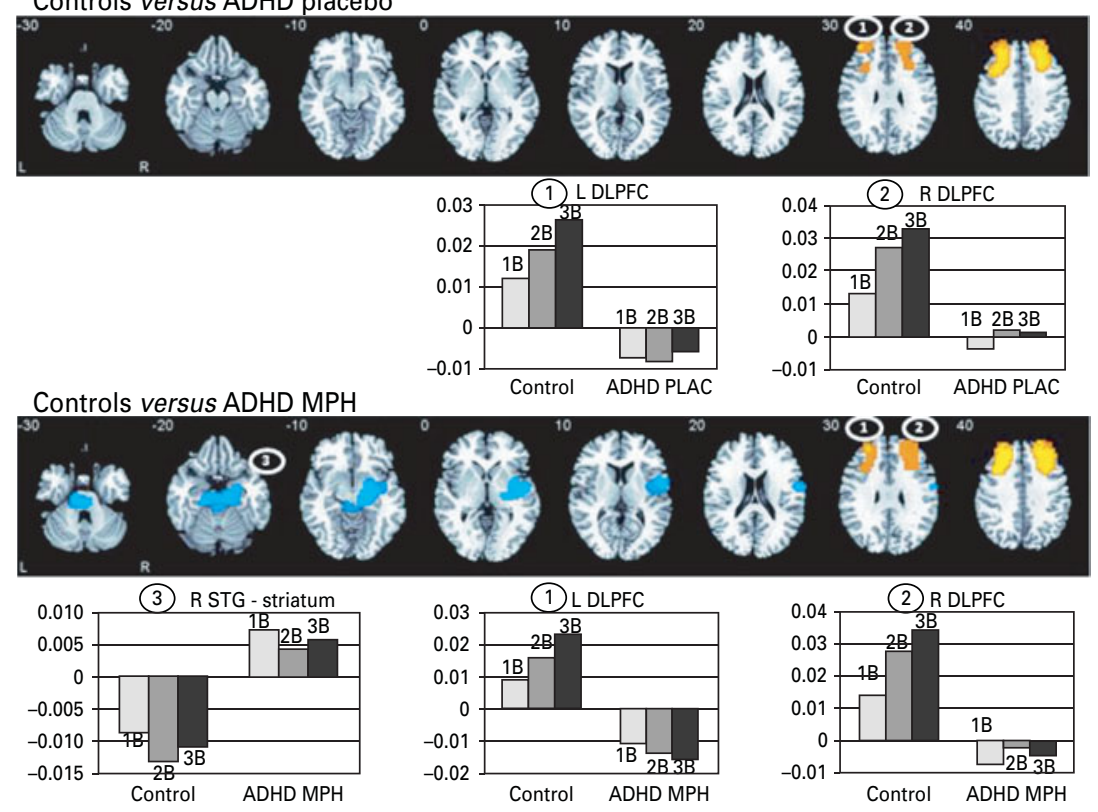

Controls versus ADHD ATX

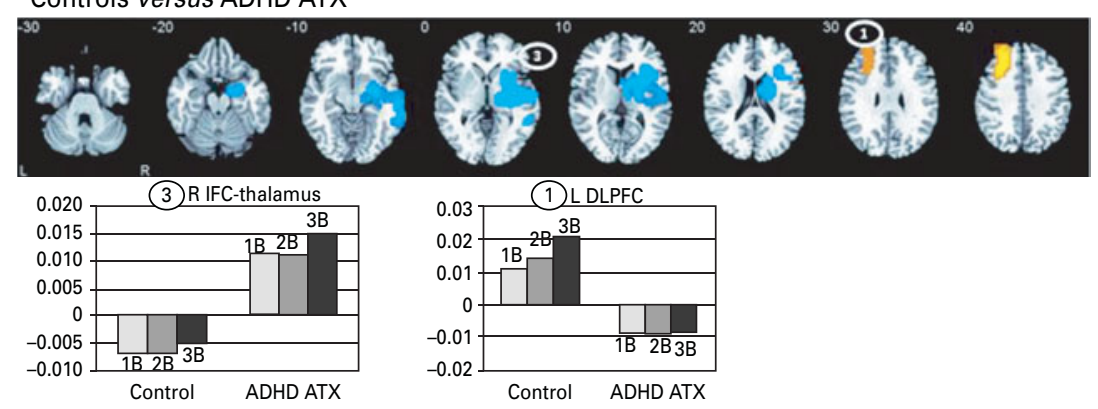

(b)

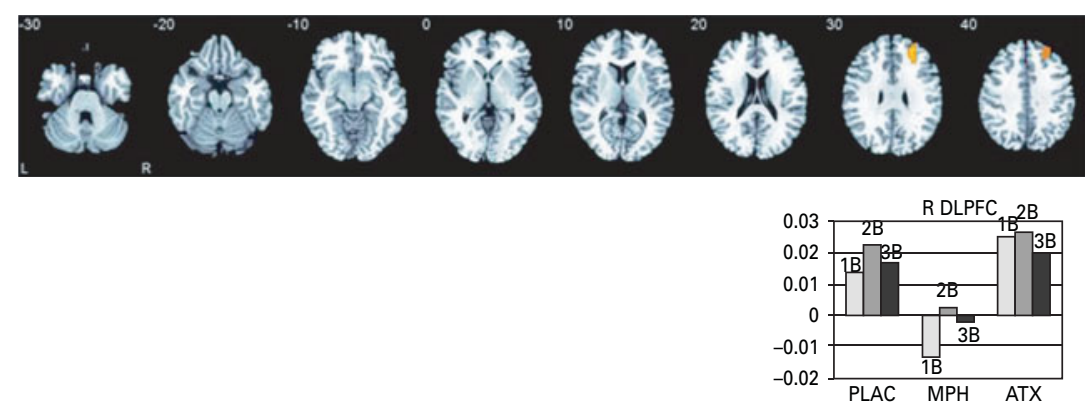

Fig. 1. Results from the main group comparisons and the within-patient drug condition analysis. (a) Between-group analysis of variance (ANOVA) comparisons between healthy control boys and boys with attention deficit hyperactivity disorder (ADHD) under either placebo (PLAC), methylphenidate (MPH) or atomoxetine (ATX) (main effect of group differences): axial sections showing the ANOVA between-group differences in brain activation between healthy control boys and boys with ADHD under each drug condition (placebo, MPH, ATX). For ease of view, clusters and their correspondent bar chart are numbered. Clusters in orange denote areas where control boys showed enhanced activation compared with ADHD boys, clusters in blue denote areas where ADHD boys showed enhanced activation compared with control boys. The graphs show the statistical measure of blood oxygen level-dependent (BOLD) (brain activation) response in each area for each group and working memory (WM) condition. The $\mathrm{x}$-axis of the graphs corresponds to the statistical measure of the BOLD response in this region. In the bar charts, the labels $1 \mathrm{~B}, 2 \mathrm{~B}$ and $3 \mathrm{~B}$ denote the BOLD response of each group in the contrasts 1-back $v$. 0-back, 2-back $v$. 0-back and 3-back $v$. 0-back, respectively. (b) Within-patients ANOVA comparison (main effect of drug condition). Axial sections showing the repeated-measures ANOVA results of drug condition within ADHD boys. The graphs 
were observed in bilateral occipital regions extending to the cuneus, due to the enhanced deactivation of this cluster in patients under MPH compared with controls during 2-back ( $p<0.001)$ (Fig. 2a, Table 3).

In the comparison between controls and patients under ATX, significant interaction effects were observed in the anterior and posterior cingulate extending to the precuneus, due to significantly enhanced activation within patients relative to controls during 1-back $(p<0.004)$ and enhanced deactivation during 3-back $(p<0.005)$ (Fig 2a, Table 3).

\section{Within-subjects comparison: WM load $\times$ drug condition}

Significant interaction effects were observed in the left IFC [14 voxels, peak Talairach coordinates $(\mathrm{x}, \mathrm{y}, \mathrm{z})$ : -36, 22, 9; BA 44/45, $p<0.019 \quad(p<0.04$ Bonferroni corrected)]) (Fig. 2b), due to significantly enhanced activation in this cluster during 2-back when under MPH compared with placebo $(p<0.001)$ and ATX $(p<0.015)$.

Repeated-measures ANOVAs showed no scan order effect on the extracted BOLD response measures.

\section{Discussion}

The present fMRI comparison between single-dose challenges of MPH and ATX on neural correlates of WM in children with ADHD shows both shared as well as drug-specific laterality effects on frontal activations. In line with previous studies, medicationnaive ADHD boys under placebo relative to controls showed impaired performance under high but not low WM load. This was accompanied by placeboassociated underactivation in the left and right DLPFC relative to controls. Both drugs had no effect on within-subject performance, but normalized the case-control performance deficits in the more difficult 2- and 3-back conditions in ADHD patients relative to controls, which were no longer observed under either drug. ATX had drug-specific modulation effects on the right DLPFC, which was upregulated in ADHD patients relative to $\mathrm{MPH}$ and significantly normalized in its underactivation relative to controls. By contrast, $\mathrm{MPH}$ showed a drug-specific WM load-dependent effect on the left-lateralized IFC, which was upregulated during 2-back, both relative to controls (case-control comparison), and relative to ATX and placebo (withinsubject contrast). Both drugs, in addition, elicited abnormally enhanced activation in patients relative to controls in different fronto-STG-striatal networks, which were associated with improved performance, suggesting compensatory effects. The findings therefore show both drug-specific laterality effects in the upregulation of prefrontal WM regions as well as shared effects of recruiting compensatory brain areas.

ADHD patients under placebo relative to controls showed no deficits during the first easy WM task condition. However, they were less accurate than controls during the 2-back and 3-back conditions, in line with prior evidence for more pronounced deficits in ADHD patients with increased task difficulty during verbal n-back (Kobel et al. 2009) and visuo-spatial WM tasks (Goldberg et al. 2005; Gau \& Shang, 2010). Although neither drug improved performance within patients, the case-control analyses showed that both drugs normalized the between-group accuracy deficits in the 2- and 3-back condition, as they were no longer observed. The findings are in line with evidence that MPH normalizes the performance deficits shown by ADHD patients relative to controls in a verbal n-back (Kobel et al. 2009). The normalization effects suggest relatively small accuracy improvements that were probably underpowered to emerge in the withinsubject analyses.

Compared with controls, patients showed underactivation in the bilateral DLPFC, a key region for WM, involved in the storage and coding of the temporal sequence of stimuli (Owen et al. 2005). The findings extend previous evidence of underfunctioning of the DLPFC in adult ADHD during WM (Valera et al. 2010) and in ADHD children during other attention and executive function tasks (Rubia, 2011; Cubillo et al. 2012a, Hart et al. 2012; Christakou et al. 2013).

The most interesting findings are those of drugspecific lateralization effects on frontal activations. While ATX showed a drug-specific right-hemispheric DLPFC upregulation and normalization effect relative to $\mathrm{MPH}, \mathrm{MPH}$ showed a drug-specific left-lateralized WM load-dependent upregulation effect on left IFC/ DLPFC activation during the 2-back condition relative to ATX within patients and relative to controls.

show the statistical measure of BOLD response in the cluster for drug condition and WM load condition. The $\mathrm{x}$-axis of the graphs corresponds to the statistical measure of the BOLD response in this region. In the bar charts, the labels $1 \mathrm{~B}, 2 \mathrm{~B}$ and $3 \mathrm{~B}$ denote the BOLD response of each group in the contrasts 1-back $v$. 0-back, 2-back $v$. 0-back and 3-back $v$. 0-back, respectively. Talairach z-coordinates are indicated for slice distance (in $\mathrm{mm}$ ) from the intercommissural line. The right side of the image corresponds to the right side of the brain. The maps are thresholded to give less than one type I error three-dimensional cluster per map $(p<0.01)$. L, Left; DLPFC, dorsolateral prefrontal cortex; R, right; STG, superior temporal gyrus; IFC, inferior frontal cortex. 
(a)

Controls versus ADHD under MPH
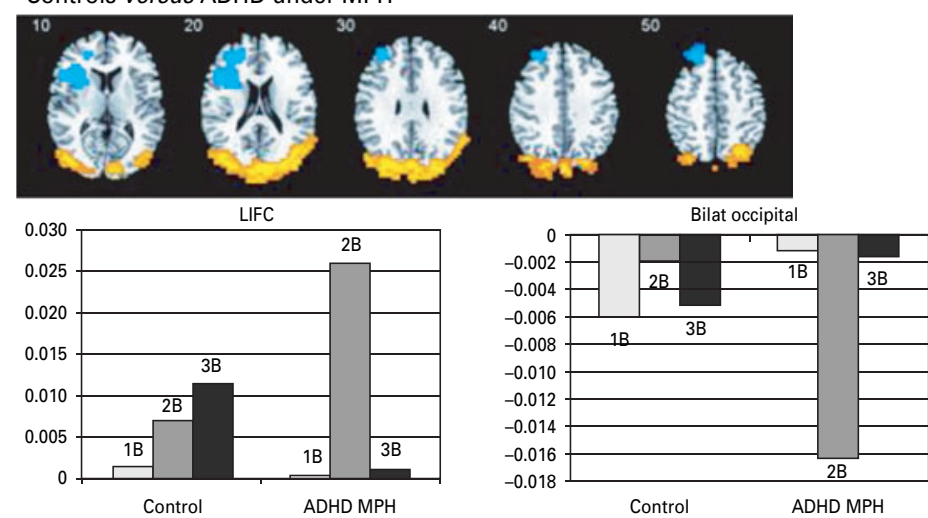

Controls versus ADHD under ATX
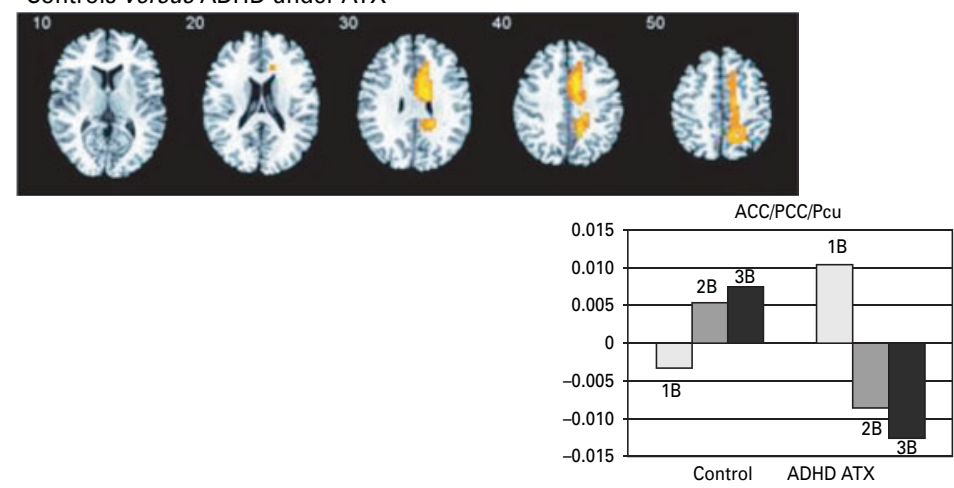

(b)
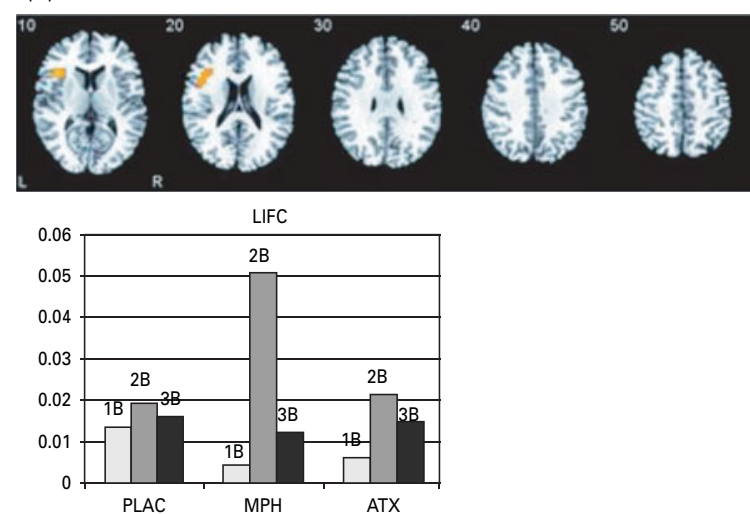

Fig. 2. Interaction effects with working memory (WM) load. (a) Axial sections showing the interaction effects between WM load and group for the between-group comparisons between healthy controls and boys with attention deficit hyperactivity disorder (ADHD) under methylphenidate (MPH), and between healthy controls and ADHD boys under atomoxetine (ATX). The graphs show the blood oxygen level-dependent (BOLD) response in each area for each group and WM load condition. In the bar charts, the labels $1 \mathrm{~B}, 2 \mathrm{~B}$ and $3 \mathrm{~B}$ denote the BOLD response of each group in the contrasts 1-back $v$. 0-back, 2-back $v$. 0 -back and 3-back $v$. 0-back, respectively. Clusters in orange denote areas where control boys showed enhanced activation compared with ADHD boys, clusters in blue denote areas where ADHD boys showed enhanced activation compared with control boys. (b) Axial section showing the interaction effects of WM load and drug condition for the within-ADHD ANOVA. The graph shows the BOLD response in each area for each drug condition during each WM load condition. In the bar charts, the labels 1B, 2B and 3B denote the BOLD response of each group in the contrasts 1-back v. 0-back, 2-back v. 0-back and 3-back $v$. 0-back, respectively. Talairach z-coordinates are indicated for slice distance (in $\mathrm{mm}$ ) from the intercommissural line. The right side of the image corresponds to the right side of the brain. The maps are thresholded to give less than one type I error three-dimensional cluster per map $(p<0.01)$. L, Left; IFC, inferior frontal cortex; Bilat, bilateral; ACC/PCC/Pcu, anterior cingulate/posterior cingulate cortex/precuneus; PLAC, placebo. 
Table 2. Group differences in brain activation for between-group ANOVA comparisons between controls and boys with ADHD under either the placebo, MPH or ATX condition ${ }^{\mathrm{a}}$

\begin{tabular}{|c|c|c|c|c|c|c|}
\hline Subject contrast & Brain regions of activation & $\begin{array}{l}\text { Brodmann } \\
\text { area }\end{array}$ & $\begin{array}{l}\text { Peak Talairach } \\
\text { coordinates: } \\
\mathrm{x}, \mathrm{y}, \mathrm{z}\end{array}$ & $\begin{array}{l}\text { Voxel } \\
\text { number }\end{array}$ & $\begin{array}{l}\text { Cluster } \\
p \text { value } \\
\text { (corrected) }\end{array}$ & Cohen's $d$ \\
\hline \multirow{2}{*}{$\begin{array}{l}\text { Control }>\text { ADHD } \\
\text { placebo }\end{array}$} & Right medial/superior frontal & $9 / 8$ & $25,37,31$ & 129 & $0.01(0.02)$ & 1.28 \\
\hline & Left medial/superior/inferior frontal & $9 / 8$ & $-25,44,31$ & 129 & $0.008(0.016)$ & 1.51 \\
\hline Control>ADHD & Right medial/superior frontal & $9 / 8$ & $25,44,31$ & 201 & $0.001(0.002)$ & 1.90 \\
\hline $\mathrm{MPH}$ & Left medial/superior frontal & $9 / 8$ & $-25,44,31$ & 143 & $0.002(0.004)$ & 1.73 \\
\hline $\begin{array}{l}\text { ADHD } \\
\text { MPH>control }\end{array}$ & $\begin{array}{l}\text { Right superior temporal/ } \\
\text { parahippocampal gyri/premotor } \\
\text { cortex/basal ganglia/thalamus/insula/ } \\
\text { amygdala/vermis cerebellum }\end{array}$ & $36 / 22 / 6$ & $18,-15,-24$ & 297 & $0.005(0.01)$ & 1.60 \\
\hline $\begin{array}{l}\text { Control>ADHD } \\
\text { ATX }\end{array}$ & Left medial/superior frontal & $9 / 8$ & $-25,41,37$ & 169 & $0.004(0.008)$ & 1.65 \\
\hline $\begin{array}{l}\text { ADHD } \\
\text { ATX>control }\end{array}$ & $\begin{array}{l}\text { Right inferior frontal/insula/medial/ } \\
\text { superior temporal/amygdala/basal } \\
\text { ganglia/thalamus }\end{array}$ & $44 / 45 / 21 / 22$ & $29,-15,-7$ & 395 & $0.003(0.006)$ & 1.82 \\
\hline
\end{tabular}

ANOVA, Analysis of variance; ADHD, attention deficit hyperactivity disorder; MPH, methylphenidate; ATX, atomoxetine.

${ }^{a}$ The maps are thresholded to give less than one type I error three-dimensional cluster per map.

Table 3. Group $\times$ WM load interaction effects on brain activation for between-group ANOVA comparisons between controls and boys with ADHD under either the placebo, methylphenidate or atomoxetine condition ${ }^{\text {a }}$

\begin{tabular}{|c|c|c|c|c|c|c|}
\hline Subject contrast & $\begin{array}{l}\text { Brain regions } \\
\text { of activation }\end{array}$ & $\begin{array}{l}\text { Brodmann } \\
\text { area }\end{array}$ & $\begin{array}{l}\text { Peak Talairach } \\
\text { coordinates: } \\
\mathrm{x}, \mathrm{y}, \mathrm{z}\end{array}$ & $\begin{array}{l}\text { Voxel } \\
\text { number }\end{array}$ & $\begin{array}{l}\text { Cluster } \\
p \text { value } \\
\text { (corrected) }\end{array}$ & Cohen's $d$ \\
\hline $\begin{array}{l}\text { ADHD MPH> } \\
\text { control }\end{array}$ & $\begin{array}{l}\text { Left inferior/middle/ } \\
\text { superior frontal/putamen }\end{array}$ & $44 / 45 / 10 / 46 / 9$ & $-36,22,4$ & 237 & $0.007(0.014)$ & 0.85 \\
\hline $\begin{array}{l}\text { Control>ADHD } \\
\mathrm{MPH}\end{array}$ & $\begin{array}{l}\text { Right and left medial } \\
\text { occipital/cuneus/middle } \\
\text { temporal gyri }\end{array}$ & $17 / 18 / 19 / 39$ & $-25,-85,20$ & 546 & $0.001(0.002)$ & 1.18 \\
\hline $\begin{array}{l}\text { Control>ADHD } \\
\text { ATX }\end{array}$ & $\begin{array}{l}\text { Right anterior/posterior } \\
\text { cingulate gyri/precuneus }\end{array}$ & $24 / 32 / 23 / 31 / 7$ & $11,15,26$ & 167 & $0.004(0.008)$ & 0.98 \\
\hline
\end{tabular}

WM, Working memory; ANOVA, analysis of variance; ADHD, attention deficit hyperactivity disorder; MPH, methylphenidate; ATX, atomoxetine.

${ }^{a}$ The maps are thresholded to give less than one type I error three-dimensional cluster per map.

The findings suggest potential differences in their effects on underlying catecholaminergic systems. WM is mediated in the DLPFC by noradrenergic $\alpha_{2}$ receptors that increase neural 'signalling' (increased firing to relevant stimuli) and by dopaminergic $D_{1}$ receptors that decrease 'noise' (suppressing firing to irrelevant stimuli) (Gamo et al. 2010). Although ATX affects both DA and NE in the PFC (Bymaster et al. 2002), in non-human primates it increases 'signalling' more frequently than it decreases 'noise' (Gamo et al. 2010). This study thus shows for the first time that upregulation of right frontolateral activation is not only observed with a single dose of ATX in healthy adults
(Chamberlain et al. 2009; Graf et al. 2011), but also in children with ADHD, implying similar mechanisms of action in both healthy subjects and ADHD patients. Most importantly, however, we show that, during $\mathrm{WM}$, the right frontal normalization and upregulation effects with ATX are drug-specific relative to MPH.

Interestingly, the finding of left IFC upregulation under the 2-back with MPH reflects the association between dopaminergic striatal function and left IFC activation during WM maintenance (Landau et al. 2009), in line with previous findings of predominantly left-hemispheric upregulation and normalization effects (Epstein et al. 2007; Rubia et al. 2009a, 2011b). 
Therefore, the findings could possibly suggest stronger effects of ATX on predominantly right-lateralized NE-mediated WM and attention networks (Tucker \& Williamson, 1984), while MPH showed stronger effects on predominantly left-lateralized fronto-striatal DA systems (Glick et al. 1982; Flor-Henry, 1986).

The enhanced deactivation with both drugs in regions of the DMN in ADHD relative to controls during the high WM load conditions suggest that catecholamine reuptake inhibitors not only increase task-positive but also switch off task-negative DMN activation. The findings extend prior evidence of enhanced DMN deactivation with MPH during motor inhibition in previously medicated patients relative to an off-medication condition (Liddle et al. 2010) to medication-naive patients in a placebo-controlled design for another task and furthermore show for the first time that this effect is shared by ATX.

Both drugs showed abnormally enhanced frontoSTG-striatal activation in ADHD boys relative to controls, although in somewhat different locations, affecting the premotor cortex with $\mathrm{MPH}$ and the right IFC with ATX. The findings echo previous findings of fronto-temporo-striatal upregulation/normalization in ADHD children with MPH during WM (PrehnKristensen et al. 2011) and other cognitive control tasks (Vaidya et al. 1998; Epstein et al. 2007; Rubia et al. 2011a,b) and extend for the first time previous evidence of enhanced IFC/STG activation after a singledose ATX challenge in healthy adults (Chamberlain et al. 2009) to a paediatric ADHD group.

The strength of the study is the double-blind, placebo-controlled design and the recruitment of medication-naive children with ADHD, which avoids the confound of a previous history of stimulant medication (Nakao et al. 2011; Hart et al. 2012).

A limitation is the fact that groups differed in IQ. However, the findings survived when outliers were excluded from the analyses.

An important caveat is that while $\mathrm{MPH}$ has an immediate effect on ADHD symptoms (Greenhill et al. 2001), ATX reaches its maximum clinical efficacy after 12 weeks of treatment (Montoya et al. 2009). Given the differences of the two drugs in temporal courses to clinical efficacy, however, future studies should also compare their long-term effects on brain activation when they have reached maximum clinical efficacy. Also, while a within-group design was crucial to assess differential drug effects, it has the disadvantage that only one group was medication-naive in the second drug and none in the third drug condition. However, the 7 days' break between scans should have minimized carry-over effects. MPH has a $3.9 \mathrm{~h}$ half-life (Modi et al. 2000). For ATX, the plasma half-life is $3 \mathrm{~h}$ for fast metabolizers and $16-25 \mathrm{~h}$ for poor metabolizers (single dose and steady state) (Sauer et al. 2003; Witcher et al. 2003) and hence effects should have been washed out after 1 week. While we used the smallest clinical dosages with minimal side effects at the time of the study as recommended by the NICE guidelines (National Institute for Heath and Clinical Excellence, 2008), some children with ADHD receive higher dosages in clinical practice, which could lead to more pronounced effects and long-term changes in catecholaminergic systems. A further limitation is that ADHD boys performed the task three times, while, for financial and ethical reasons, controls were scanned only once, unmedicated. However, the counterbalanced randomized design for the ADHD group adequately controlled for practice effects in patients, and we found no order effects for the within or the case-control studies, which suggests that these were unlikely to have confounded the between-groups analyses. Also, we included a relatively large age range. While this does not affect the within-subject analyses and controls were age-matched, a smaller age range may have resulted in less variance and more refined changes particular to specific ages, given developmental changes between late childhood and adolescence during WM and other executive functions (Crone et al. 2006; Bunge \& Wright, 2007; Rubia, 2012). Furthermore, due to the higher prevalence of ADHD in boys (Merikangas et al. 2010), we only tested male adolescents, which limits the generalizability of the findings.

In conclusion, the findings show both drug-specific as well as shared effects on task-positive and tasknegative WM networks. ATX appears to have a drugspecific effect of upregulating and normalizing WM-related right DLPFC dysfunction in ADHD, while $\mathrm{MPH}$ appears to upregulate compensatory activation of left IFC activation, but only during the 2-back condition.

\section{Supplementary material}

For supplementary material accompanying this paper visit http://dx.doi.org/10.1017/S0033291713000676

\section{Acknowledgements}

The study was funded by the Department of Health via the National Institute for Health Research Biomedical Research Centre (NIHR BRC) for Mental Health at the South London and Maudsley NHS Foundation Trust and Institute of Psychiatry, King's College London and Lilly Pharmaceuticals. The NIHR BRC also supported A.C. and A.B.S. via a Ph.D. studentship and post-doctoral fellowship, respectively. 


\section{Declaration of Interest}

K.R. has received speaker's honoraria from Shire, Novartis, Medice and Lilly. Lilly had no input into the design of the study, its analysis or interpretation.

\section{References}

APA (2000). Diagnostic and Statistical Manual of Mental Disorders, 4th edn, Text Revision. American Psychiatric Association: Washington, DC.

Baddeley A (1996). The fractionation of working memory. Proceedings of the National Academy of Sciences USA 93, 13468-13472.

Brain Image Analysis Unit (2011). XBAM (http://www. brainmap.co.uk). Accessed 24 April 2012.

Brammer MJ, Bullmore ET, Simmons A, Williams SC, Grasby PM, Howard RJ, Woodruff PW, Rabe-Hesketh S (1997). Generic brain activation mapping in functional magnetic resonance imaging: a nonparametric approach. Magnetic Resonance Imaging 15, 763-770.

Bridgett DJ, Walker ME (2006). Intellectual functioning in adults with ADHD: a meta-analytic examination of full scale IQ differences between adults with and without ADHD. Psychological Assessment 18, 1-14.

Bullmore E, Long C, Suckling J, Fadili J, Calvert G, Zelaya F, Carpenter TA, Brammer M (2001). Colored noise and computational inference in neurophysiological (fMRI) time series analysis: resampling methods in time and wavelet domains. Human Brain Mapping 12, 61-78.

Bullmore ET, Brammer MJ, Rabe-Hesketh S, Curtis VA, Morris RG, Williams SC, Sharma T, McGuire PK (1999). Methods for diagnosis and treatment of stimulus-correlated motion in generic brain activation studies using fMRI. Human Brain Mapping 7, 38-48.

Bunge SA, Wright SB (2007). Neurodevelopmental changes in working memory and cognitive control. Current Opinion in Neurobiology 17, 243-250.

Bymaster FP, Katner JS, Nelson DL, Hemrick-Luecke SK, Threlkeld PG, Heiligenstein JH, Morin SM, Gehlert DR, Perry KW (2002). Atomoxetine increases extracellular levels of norepinephrine and dopamine in prefrontal cortex of rat: a potential mechanism for efficacy in attention deficit/ hyperactivity disorder. Neuropsychopharmacology 27, 699-711.

Chamberlain SR, Del Campo N, Dowson J, Muller U, Clark L, Robbins TW, Sahakian BJ (2007). Atomoxetine improved response inhibition in adults with attention deficit/hyperactivity disorder. Biological Psychiatry 62, 977-984.

Chamberlain SR, Hampshire A, Muller U, Rubia K, Del Campo N, Craig K, Regenthal R, Suckling J, Roiser JP, Grant JE, Bullmore ET, Robbins TW, Sahakian BJ (2009). Atomoxetine modulates right inferior frontal activation during inhibitory control: a pharmacological functional magnetic resonance imaging study. Biological Psychiatry 65, 550-555.
Chan YP, Swanson JM, Soldin SS, Thiessen JJ, Macleod SM, Logan W (1983). Methylphenidate hydrochloride given with or before breakfast: II. Effects on plasma concentration of methylphenidate and ritalinic acid. Pediatrics 72, 56-59.

Christakou A, Murphy CM, Chantiluke K, Cubillo AI, Smith AB, Giampietro V, Daly E, Ecker C, Robertson D; MRC AIMS consortium, Murphy DG, Rubia K (2013). Disorder-specific functional abnormalities during sustained attention in youth with attention deficit hyperactivity disorder (ADHD) and with autism. Molecular Psychiatry 18, 236-244.

Conners CK, Sitarenios G, Parker JDA, Epstein JN (1998). Revision and restandardization of the Conners Teacher Rating Scale (CTRS-R): factor structure, reliability, and criterion validity. Journal of Abnormal Child Psychology 26, 279-291.

Crone EA, Wendelken C, Donohue S, van Leijenhorst L, Bunge SA (2006). Neurocognitive development of the ability to manipulate information in working memory. Proceedings of the National Academy of Sciences USA 103, 9315-9320.

Cubillo A, Halari R, Smith A, Taylor E, Rubia K (2012a). A review of fronto-striatal and frontocortical brain abnormalities in children and adults with attention deficit hyperactivity disorder (ADHD) and new evidence for dysfunction in adults with ADHD during motivation and attention. Cortex 48, 194-215.

Cubillo A, Smith AB, Barrett N, Giampietro V, Brammer MJ, Simmons A, Rubia K (2012b). Shared and drug-specific effects of atomoxetine and methylphenidate on inhibitory brain dysfunction in medication-naive ADHD boys. Cerebral Cortex. Published online 9 October 2012. doi:10.1093/cercor/bhs296.

Dennis M, Francis DJ, Cirino PT, Schachar R, Barnes MA, Fletcher JM (2009). Why IQ is not a covariate in cognitive studies of neurodevelopmental disorders. Journal of the International Neuropsychological Society 15, 331-343.

Epstein JN, Casey BJ, Tonev ST, Davidson MC, Reiss AL, Garrett A, Hinshaw SP, Greenhill LL, Glover G, Shafritz KM, Vitolo A, Kotler LA, Jarrett MA, Spicer J (2007). ADHD- and medication-related brain activation effects in concordantly affected parent-child dyads with ADHD. Journal of Child Psychology and Psychiatry 48, 899-913.

Evans SH, Anastasio EJ (1968). Misuse of analysis of covariance when treatment effect and covariate are confounded. Psychological Bulletin 69, 225-234.

Flor-Henry P (1986). Observations, reflections and speculations on the cerebral determinants of mood and on the bilaterally asymmetrical distributions of the major neurotransmitter systems. Acta Neurologica Scandinavica (Suppl.) 109, 75-89.

Gallezot JD, Weinzimmer D, Nabulsi N, Lin SF, Fowles K, Sandiego C, McCarthy TJ, Maguire RP, Carson RE, Ding YS (2011). Evaluation of $\left[{ }^{11} \mathrm{C}\right] \mathrm{MRB}$ for assessment of occupancy of norepinephrine transporters: studies with atomoxetine in non-human primates. Neuroimage 56, 268-279. 
Gamo NJ, Wang M, Arnsten AF (2010). Methylphenidate and atomoxetine enhance prefrontal function through $\alpha_{2}$-adrenergic and dopamine $\mathrm{D}_{1}$ receptors. Journal of the American Academy of Child and Adolescent Psychiatry 49, 1011-1023.

Gau SS, Shang CY (2010). Executive functions as endophenotypes in ADHD: evidence from the Cambridge Neuropsychological Test Battery (CANTAB). Journal of Child Psychology and Psychiatry 51, 838-849.

Ginestet CE, Simmons A (2011). Statistical parametric network analysis of functional connectivity dynamics during a working memory task. Neuroimage 55, 688-704.

Glick SD, Ross DA, Hough LB (1982). Lateral asymmetry of neurotransmitters in human brain. Brain Research 234, 53-63.

Goldberg D, Murray R (eds) (2002). Maudsley Handbook of Practical Psychiatry. Oxford University Press: Oxford.

Goldberg MC, Mostofsky SH, Cutting LE, Mahone EM, Astor BC, Denckla MB, Landa RJ (2005). Subtle executive impairment in children with autism and children with ADHD. Journal of Autism and Developmental Disorders 35, 279-293.

Goodman R, Scott S (1999). Comparing the Strengths and Difficulties Questionnaire and the Child Behavior Checklist: is small beautiful? Journal of Abnormal Child Psychology 27, $17-24$.

Graf H, Abler B, Freudenmann R, Beschoner P, Schaeffeler E, Spitzer M, Schwab M, Gron G (2011). Neural correlates of error monitoring modulated by atomoxetine in healthy volunteers. Biological Psychiatry 69, 890-897.

Greenhill LL, Swanson JM, Vitiello B, Davies M, Clevenger W, Wu M, Arnold LE, Abikoff HB, Bukstein OG, Conners CK, Elliott GR, Hechtman L, Hinshaw SP, Hoza B, Jensen PS, Kraemer HC, March JS, Newcorn JH, Severe JB, Wells K, Wigal T (2001). Impairment and deportment responses to different methylphenidate doses in children with ADHD: the MTA titration trial. Journal of the American Academy of Child and Adolescent Psychiatry 40, 180-187.

Hannestad J, Gallezot JD, Planeta-Wilson B, Lin SF, Williams WA, van Dyck CH, Malison RT, Carson RE, Ding YS (2010). Clinically relevant doses of methylphenidate significantly occupy norepinephrine transporters in humans in vivo. Biological Psychiatry 68, 854-860.

Hart H, Radua J, Mataix-Cols D, Rubia K (2012). Meta-analysis of fMRI studies of timing in attention-deficit hyperactivity disorder (ADHD). Neuroscience and Biobehavioral Reviews 36, 2248-2256.

Hart H, Radua J, Nakao T, Mataix-Cols D, Rubia K (2013). Meta-analysis of functional magnetic resonance imaging studies of inhibition and attention in attention-deficit/hyperactivity disorder: exploring task-specific, stimulant medication, and age effects. JAMA Psychiatry 70, 185-198.

Hazell PL, Kohn MR, Dickson R, Walton RJ, Granger RE, van Wyk GW (2010). Core ADHD symptom improvement with atomoxetine versus methylphenidate: a direct comparison meta-analysis. Journal of Attention Disorders $\mathbf{1 5}$ 674-683.

Klein C, Wendling K, Huettner P, Ruder H, Peper M (2006). Intra-subject variability in attention-deficit hyperactivity disorder. Biological Psychiatry 60, 1088-1097.

Kobel M, Bechtel N, Weber P, Specht K, Klarhofer M, Scheffler K, Opwis K, Penner IK (2009). Effects of methylphenidate on working memory functioning in children with attention deficit/hyperactivity disorder. European Journal of Paediatric Neurology 13, 516-523.

Lancaster JL, Rainey LH, Summerlin JL, Freitas CS, Fox PT, Evans AC, Toga AW, Mazziotta JC (1997). Automated labeling of the human brain: a preliminary report on the development and evaluation of a forward-transform method. Human Brain Mapping 5, 238-242.

Lancaster JL, Woldorff MG, Parsons LM, Liotti M, Freitas CS, Rainey L, Kochunov PV, Nickerson D, Mikiten SA, Fox PT (2000). Automated Talairach atlas labels for functional brain mapping. Human Brain Mapping 10, 120-131.

Landau SM, Lal R, O'Neil JP, Baker S, Jagust WJ (2009). Striatal dopamine and working memory. Cerebral Cortex 19, 445-454.

Liddle EB, Hollis C, Batty MJ, Groom MJ, Totman JJ, Liotti M, Scerif G, Liddle PF (2010). Task-related default mode network modulation and inhibitory control in ADHD: effects of motivation and methylphenidate. Journal of Child Psychology and Psychiatry 52, 761-771.

Lijffijt M, Kenemans JL, ter Wal A, Quik EH, Kemner C, Westenberg H, Verbaten MN, van Engeland H (2006). Dose-related effect of methylphenidate on stopping and changing in children with attention-deficit/hyperactivity disorder. European Psychiatry 21, 544-547.

Marquand AF, De Simoni S, O'Daly OG, Williams SC, Mourao-Miranda J, Mehta MA (2011). Pattern classification of working memory networks reveals differential effects of methylphenidate, atomoxetine, and placebo in healthy volunteers. Neuropsychopharmacology 36, 1237-1247.

Martinussen R, Hayden J, Hogg-Johnson S, Tannock R (2005). A meta-analysis of working memory impairments in children with attention-deficit/hyperactivity disorder. Journal of the American Academy of Child and Adolescent Psychiatry 44, 377-384.

Matthews JN, Altman DG (1996). Statistics notes. Interaction 2: compare effect sizes not $P$ values. British Medical Journal 313, 808.

Merikangas KR, He JP, Brody D, Fisher PW, Bourdon K, Koretz DS (2010). Prevalence and treatment of mental disorders among US children in the 2001-2004 NHANES. Pediatrics 125, 75-81.

Miller G, Chapman J (2001). Misunderstanding analysis of covariance. Journal of Abnormal Psychology 110, 40-48.

Modi N, Lindemulder B, Gupta S (2000). Single- and multiple-dose pharmacokinetics of an oral once-a-day osmotic controlled-release OROS (methylphenidate $\mathrm{HCl}$ ) formulation. Journal of Clinical Pharmacology 40, 379-388.

Montoya A, Hervas A, Cardo E, Artigas J, Mardomingo MJ, Alda JA, Gastaminza X, Garcia-Polavieja MJ, Gilaberte I, 
Escobar R (2009). Evaluation of atomoxetine for first-line treatment of newly diagnosed, treatment-naive children and adolescents with attention deficit/hyperactivity disorder. Current Medical Research and Opinion 25, 2745-2754.

Nakao T, Radua J, Rubia K, Mataix-Cols D (2011). Gray matter volume abnormalities in ADHD and the effects of stimulant medication: voxel-based meta-analysis. American Journal of Psychiatry 168, 1154-1163.

National Institute for Heath and Clinical Excellence (2008). Attention deficit hyperactivity disorder: diagnosis and management of ADHD in children, young people and adults (http://www.nice.org.uk/CG72). Accessed 24 April 2012.

Owen AM, McMillan KM, Laird AR, Bullmore E (2005). $\mathrm{N}$-back working memory paradigm: a meta-analysis of normative functional neuroimaging studies. Human Brain Mapping 25, 46-59.

Pasini A, Paloscia C, Alessandrelli R, Porfirio MC, Curatolo P (2007). Attention and executive functions profile in drug naive ADHD subtypes. Brain and Development 29, 400-408.

Prehn-Kristensen A, Krauel K, Hinrichs H, Fischer J, Malecki U, Schuetze H, Wolff S, Jansen O, Duezel E, Baving L (2011). Methylphenidate does not improve interference control during a working memory task in young patients with attention-deficit hyperactivity disorder. Brain Research 1388, 56-68.

Rhodes SM, Park J, Seth S, Coghill DR (2011). A comprehensive investigation of memory impairment in attention deficit hyperactivity disorder and oppositional defiant disorder. Journal of Child Psychology and Psychiatry 53, 128-137.

Rommelse NN, Altink ME, Oosterlaan J, Buschgens CJ, Buitelaar J, Sergeant JA (2008). Support for an independent familial segregation of executive and intelligence endophenotypes in ADHD families. Psychological Medicine 38, 1595-1606.

Rubia K (2011). 'Cool' inferior fronto-striatal dysfunction in attention-deficit hyperactivity disorder (ADHD) versus 'hot' ventromedial orbitofronto-limbic dysfunction in conduct disorder: a review. Biological Psychiatry 69, e69-e87.

Rubia K (2012). Functional brain imaging across development. European Child and Adolescent Psychiatry. Published online 24 June 2012. doi:10.1007/ s00787-012-0291-8.

Rubia K, Halari R, Christakou A, Taylor E (2009a). Impulsiveness as a timing disturbance: neurocognitive abnormalities in attention-deficit hyperactivity disorder during temporal processes and normalization with methylphenidate. Philosophical Transactions of the Royal Society of London. Series B, Biological Sciences 364, 1919-1931.

Rubia K, Halari R, Cubillo A, Mohammad AM, Brammer M, Taylor E (2011a). Methylphenidate normalises fronto-striatal underactivation during interference inhibition in medication-naive children with attention-deficit hyperactivity disorder. Neuropsychopharmacology 36, 1575-1586.
Rubia K, Halari R, Cubillo A, Mohammad M, Taylor E (2009b). Methylphenidate normalises activation and functional connectivity deficits in attention and motivation networks in medication-naive children with ADHD during a Rewarded Continuous Performance Task.

Neuropharmacology 57, 640-652.

Rubia K, Halari R, Mohammad AM, Taylor E, Brammer M (2011b). Methylphenidate normalises fronto-cingulate underactivation during error processing in attention-deficit hyperactivity disorder. Biological Psychiatry 70, 255-262.

Rutter M, Bailey A, Berument S, LeCouteur A, Lord C, Pickles A (eds) (2003). Social Communication Questionnaire (SCQ). Western Psychological Services: Los Angeles.

Sauer J-M, Ponsler GD, Mattiuz EL, Long AJ, Witcher JW, Thomasson HR, Desante KA (2003). Disposition and metabolic fate of atomoxetine hydrochloride: the role of CYP2D6 in human disposition and metabolism. Drug Metabolism and Disposition 31, 98-107.

Shafritz KM, Marchione KE, Gore JC, Shaywitz SE, Shaywitz BA (2004). The effects of methylphenidate on neural systems of attention in attention deficit hyperactivity disorder. American Journal of Psychiatry 161, 1990-1997.

Sheridan MA, Hinshaw S, D'Esposito M (2010). Stimulant medication and prefrontal functional connectivity during working memory in ADHD: a preliminary report. Journal of Attention Disorders 14, 69-78.

Silk T, Vance A, Rinehart N, Egan G, O’Boyle M, Bradshaw JL, Cunnington R (2005). Fronto-parietal activation in attention-deficit hyperactivity disorder, combined type: functional magnetic resonance imaging study. British Journal of Psychiatry 187, 282-283.

Simmons A, Moore E, Williams SC (1999). Quality control for functional magnetic resonance imaging using automated data analysis and Shewhart charting. Magnetic Resonance in Medicine 41, 1274-1278.

Toplak M, Jain U, Rosemary T (2008). Executive and motivational processes in adolescents with attention-deficit-hyperactivity disorder (ADHD). Behavioural and Brain Functions 1, 8.

Tucker DM, Williamson PA (1984). Asymmetric neural control systems in human self-regulation. Psychological Review 91, 185-215.

Vaidya CJ, Austin G, Kirkorian G, Ridlehuber HW, Desmond JE, Glover GH, Gabrieli JDE (1998). Selective effects of methylphenidate in attention deficit hyperactivity disorder: a functional magnetic resonance study. Proceedings of the National Academy of Sciences USA 95, 14494-14499.

Valera EM, Brown A, Biederman J, Faraone SV, Makris N, Monuteaux MC, Whitfield-Gabrieli S, Vitulano M, Schiller M, Seidman LJ (2010). Sex differences in the functional neuroanatomy of working memory in adults with ADHD. American Journal of Psychiatry 167, 87-94.

Vance A, Silk TJ, Casey M, Rinehart NJ, Bradshaw JL, Bellgrove MA, Cunnington R (2007). Right parietal dysfunction in children with attention deficit hyperactivity disorder, combined type: a functional MRI study. Molecular Psychiatry 12, 826-832. 
Volkow ND, Wang GJ, Fowler JS, Gatley SJ, Logan J, Ding YS, Hitzemann R, Pappas N (1998). Dopamine transporter occupancies in the human brain induced by therapeutic doses of oral methylphenidate. American Journal of Psychiatry 155, 1325-1331.

Wechsler D (1999). Wechsler Abbreviated Scale of Intelligence. Psychological Corp.: San Antonio, TX.

Wechsler D (2004). Wechsler Intelligence Scale for Children, 4th edn. Psychological Corp.: London, UK.

Willcutt EG, Doyle AE, Nigg JT, Faraone SV, Pennington BF (2005). Validity of the executive function theory of attention-deficit/hyperactivity disorder: a meta-analytic review. Biological Psychiatry 57, 1336-1346.

Witcher JW, Long A, Smith B, Sauer JM, Heilgenstein J, Wilens T, Spencer T, Biederman J (2003). Atomoxetine pharmacokinetics in children and adolescents with attention deficit hyperactivity disorder. Journal of Child and Adolescent Psychopharmacology 13, 53-63.

Wong CG, Stevens MC (2012). The effects of stimulant medication on working memory functional connectivity in attention-deficit/hyperactivity disorder. Biological Psychiatry 71, 458-466. 